\title{
Theory of two-dimensional photonic crystals with lamellar cylindrical pores
}

\author{
A.E. Glushko, E.Ya. Glushko, and L.A. Karachevtseva \\ V. Lashkaryov Institute of Semiconductor Physics, NAS of Ukraine, 45, prospect Nauky, 03028 Kyiv, Ukraine \\ Phone: 525-98-15 \\ E-mail:a_glushko@ukr.net
}

\begin{abstract}
Theoretical investigation of $2 \mathrm{D}$ photonic crystals containing intermediate layers on the surface of cylindrical pores is performed within the framework of the plane-wave model. We have analyzed how the third medium introduction influences on the absolute bandgap formation in the triangular lattice photonic structure. We have obtained the dependences of the bandgap width and position on the additional interlayer thickness $d$ and its dielectric constant $\varepsilon_{i}$. The new concept of photonic contrast is introduced in order to describe the bandgap formation conditions. We conclude that addition of the surface layer decreases the photonic band gap width of the examined system.
\end{abstract}

Keywords: 2D photonic crystal, lamellar pores, photonic contrast, band structure.

Manuscript received 10.02.05; accepted for publication 18.05.05.

\section{Introduction}

The latter decade progress in new materials technology led to creation of photonic crystals that represent the class of artificial materials with periodic dielectric function $[1,2]$. The simplest case of these periodic layered structures is one-dimensional photonic crystals. The first photonic crystal had the dimensions of several centimeters and worked in a microwave range [1]. After that, many various types of $2 \mathrm{D}$ and $3 \mathrm{D}$ photonic crystals were obtained and various experimental technologies were elaborated to produce the photonic structures [3]. The photonic crystals of all types and dimensions can exhibit strong interference suppression of the electromagnetic field over the separated frequency intervals. The frequency ranges are called as photonic gaps by analogy with the electron waves in crystals [112]. The frequency intervals adjacent to gaps, i.e., photonic bands, are characterized by the constructive interference of electromagnetic waves. Therefore, if the electromagnetic wave frequency matches the photonic gap, this wave will be totally reflected from the photonic crystal. While the similar wave irradiated by an internal source will be suppressed. The unusual properties of photonic crystals provide new ways to manipulate light and varied applications in high-efficiency lasers, antennas, and all-optical devices.

First theoretical reports on solving the problem of electromagnetic wave propagation in periodic structures did not take into account the vector nature of electromagnetic field and used the scalar approximation $[2,6]$. For the first time, the full-vector calculations were carried out for 3D photonic crystals with face-centeredcubic [7, 8] and diamond [4] superlattices using the Fourier representation of Maxwell's equations. Such approach called as the plane-wave method enables to obtain numerically the dispersion law of the system, i.e., the photonic band structure.

Here, we present the theoretical investigation of $2 \mathrm{D}$ photonic crystals containing intermediate layers on the surface of cylindrical pores. The bandgap structure is calculated for various sizes of the lamellar pore walls in a triangular-lattice photonic crystal, and the problem of optimal parameters corresponding for the maximal absolute gap is discussed. The plane-wave method accuracy is evaluated by the Richardson criterion.

\section{The plane-wave method. Numerical approach}

For the first time, the plane-wave method came into the use when studying $2 \mathrm{D}$ photonic crystals in $[5,9]$. It was shown that 2D dielectric medium with air pores which form the superlattice of triangular symmetry has a wide gap in the energy spectrum. The propagation of electromagnetic waves along the direction perpendicular to the pores was considered, and it was found that the propagation is forbidden for both TE- and THpolarizations inside the energy gaps. Such zones of forbidden energy were called as an absolute or full photonic gap. The similar calculations for electromagnetic waves passing in directions nonperpendicular to pore axes were carried out in [10]. 2D photonic crystal consisting of alumina-ceramic rods arranged in a regular square lattice was investigated 


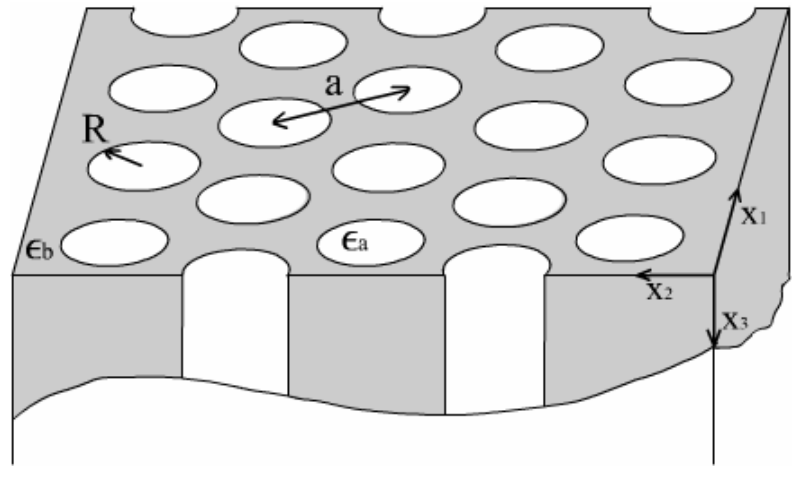

Fig. 1. The typical two-dimensional photonic crystal. Triangular lattice. $R$ - the pore radius; $a$ - the lattice constant; $\varepsilon_{a}, \varepsilon_{b}-$ the dielectric constants of the pore and the rest of medium, respectively.

experimentally using the coherent microwave transient spectroscopy [11]. The experimental results were compared to the theoretical predictions obtained using the plane-wave expansion technique $[5,9]$.

We consider the 2D macroporous photonic structure (Fig. 1) that is presented by a dielectric medium with regular air pores of circular cross-section. This structure is assumed to be infinite in all directions. The translation invariance of the system in $X_{3}$ direction and periodicity of the one in $X_{1} X_{2}$ plane set the form of the field components

$$
E_{\alpha}(\vec{x}, \omega)=E_{\alpha}\left(\vec{x}_{I I}, \omega\right) \exp \left(i k_{3} x_{3}\right), \quad \alpha=1,2,3,
$$

and dielectric function

$\varepsilon\left(\vec{x}_{I I}+\vec{x}_{I I}(l)\right)=\varepsilon\left(\vec{x}_{I I}\right)$,

where $\vec{x}_{I I}=\vec{e}_{1} x_{1}+\vec{e}_{2} x_{2}$ is a radius-vector in $X_{1} X_{2}$ plane, $\vec{x}_{I I}(l)=l_{1} \vec{a}_{1}+l_{2} \vec{a}_{2}$ are the lattice point of the photonic superlattice. The points of the lattice reciprocal to this one are given by the vectors

$$
\vec{G}_{I I}(h)=h_{1} \vec{b}_{1}+h_{2} \vec{b}_{2} \text {, }
$$

where the primitive translation vectors $\vec{b}_{1}$ and $\vec{b}_{2}$ are defined by the equations

$$
\vec{a}_{i} \vec{b}_{j}=2 \pi \delta_{i j}, i, j=1,2 \text {, }
$$

while $h_{1}, h_{2}, l_{1}, l_{2}$ run over all the positive and negative integers and zero.

After substitution (1) and (2) into Maxwell's equations, we obtain the following system:

$$
\begin{aligned}
& \frac{1}{\varepsilon\left(\vec{x}_{I I}\right)}\left(-\frac{\partial^{2} E_{1}}{\partial x_{2}^{2}}+k_{3}^{2} E_{1}+\frac{\partial^{2} E_{2}}{\partial x_{1} \partial x_{2}}+i k_{3} \frac{\partial E_{3}}{\partial x_{1}}\right)=\frac{\omega^{2}}{c^{2}} E_{1} \\
& \frac{1}{\varepsilon\left(\vec{x}_{I I}\right)}\left(\frac{\partial^{2} E_{1}}{\partial x_{1} \partial x_{2}}-\frac{\partial^{2} E_{2}}{\partial x_{1}^{2}}+k_{3}^{2} E_{2}+i k_{3} \frac{\partial E_{3}}{\partial x_{2}}\right)=\frac{\omega^{2}}{c^{2}} E_{2} \\
& \frac{1}{\varepsilon\left(\vec{x}_{I I}\right)}\left(i k_{3} \frac{\partial E_{1}}{\partial x_{1}}+i k_{3} \frac{\partial E_{2}}{\partial x_{2}}-\frac{\partial^{2} E_{3}}{\partial x_{1}^{2}}-\frac{\partial^{2} E_{3}}{\partial x_{2}^{2}}\right)=\frac{\omega^{2}}{c^{2}} E_{3} .
\end{aligned}
$$

To solve the equations (5), we expand the periodic inverse dielectric function $\varepsilon^{-1}\left(\vec{x}_{I I}\right)$

$$
\frac{1}{\varepsilon\left(\vec{x}_{I I}\right)}=\sum_{\vec{G}_{I I}} \kappa\left(\vec{G}_{I I}\right) \exp \left(i \vec{G}_{I I} \vec{x}_{I I}\right)
$$

and components of the electric field $E_{\alpha}\left(\vec{x}_{I I}, \omega\right)$,

$E_{\alpha}(\vec{x}, \omega)=\sum_{\vec{G}_{I I}} a_{\alpha}\left(\vec{k}_{I I}+\vec{G}_{I I}\right) \exp \left[i\left(\vec{k}_{I I}+\vec{G}_{I I}\right) \cdot \vec{x}_{I I}\right]$,

$\alpha=1,2,3$

into the Fourier series. The forms (6) and (7) satisfy the Bloch - Flouqet theorem, required by the 2D periodicity of the system under consideration. Here, $\vec{k}_{I I}=\vec{x}_{1} k_{1}+\vec{x}_{2} k_{2}$ is the projection of the wavevector onto the $X_{1} X_{2}$ plane.

Following to [10] after the substitution of (6) and (7) into the system (5), we obtain a system of $3 N$ equations, where $N$ is the number of basis reciprocal vectors:

$$
\begin{aligned}
& \sum_{G_{I I}^{\prime}} \widehat{\kappa}\left(\vec{G}_{I I}-\vec{G}_{I I}^{\prime}\right)\left\{\begin{array}{l}
\left(\left(k_{2}+G_{2}^{\prime}\right)^{2}+k_{3}^{2}\right) \cdot a_{1}\left(\vec{k}_{I I}+\vec{G}_{I I}^{\prime}\right)- \\
-\left(k_{1}+G_{1}^{\prime}\right)\left(k_{2}+G_{2}^{\prime}\right) \cdot a_{2}\left(\vec{k}_{I I}+\vec{G}_{I I}^{\prime}\right)- \\
-\left(k_{1}+G_{1}^{\prime}\right) k_{3} \cdot a_{3}\left(\vec{k}_{I I}+\vec{G}_{I I}^{\prime}\right)
\end{array}\right\}= \\
& =\frac{\omega^{2}}{c^{2}} a_{1}\left(\vec{k}_{I I}+\vec{G}_{I I}\right) \\
& \sum_{G_{I I}^{\prime}} \hat{\kappa}\left(\vec{G}_{I I}-\vec{G}_{I I}^{\prime}\right)\left\{\begin{array}{l}
-\left(k_{1}+G_{1}^{\prime}\right)\left(k_{2}+G_{2}^{\prime}\right) \cdot a_{1}\left(\vec{k}_{I I}+\vec{G}_{I I}^{\prime}\right)+ \\
+\left[\left(k_{1}+G_{1}^{\prime}\right)^{2}+k_{3}^{2}\right] \cdot a_{2}\left(\vec{k}_{I I}+\vec{G}_{I I}^{\prime}\right)- \\
-\left(k_{2}+G_{2}^{\prime}\right) k_{3} \cdot a_{3}\left(\vec{k}_{I I}+\vec{G}_{I I}^{\prime}\right)
\end{array}\right\}= \\
& =\frac{\omega^{2}}{c^{2}} a_{2}\left(\vec{k}_{I I}+\vec{G}_{I I}\right) \\
& \sum_{G_{I I}^{\prime}} \widehat{\kappa}\left(\vec{G}_{I I}-\vec{G}_{I I}^{\prime}\right)\left\{\begin{array}{l}
-\left(k_{1}+G_{1}^{\prime}\right) k_{3} \cdot a_{1}\left(\vec{k}_{I I}+\vec{G}_{I I}^{\prime}\right)- \\
-\left(k_{2}+G_{2}^{\prime}\right) k_{3} \cdot a_{2}\left(\vec{k}_{I I}+\vec{G}_{I I}^{\prime}\right)+ \\
+\left(\left(k_{2}+G_{2}^{\prime}\right)^{2}+\left(k_{1}+G_{1}^{\prime}\right)^{2}\right) \cdot a_{3}\left(\vec{k}_{I I}+\vec{G}_{I I}^{\prime}\right)
\end{array}\right\}= \\
& =\frac{\omega^{2}}{c^{2}} a_{3}\left(\vec{k}_{I I}+\vec{G}_{I I}\right) .
\end{aligned}
$$

The Fourier coefficients of $\varepsilon^{-1}\left(\vec{x}_{I I}\right)$ were found for triangular lattice of cylindrical pores in [10]

$$
\hat{\kappa}\left(\vec{G}_{I I}\right)= \begin{cases}f \frac{1}{\varepsilon_{a}}+(1-f) \frac{1}{\varepsilon_{b}}, & \vec{G}_{I I}=0 \\ \left(\frac{1}{\varepsilon_{a}}-\frac{1}{\varepsilon_{b}}\right) f \frac{2 J_{1}\left(G_{I I} R\right)}{G_{I I} R}, & \vec{G}_{I I} \neq 0\end{cases}
$$

where $J_{1}(x)$ is the Bessel function, and $f=\frac{2 \pi}{\sqrt{3}} \frac{R^{2}}{a^{2}}$ is the filling fraction, i. e., the fraction of the total volume occupied by cylinder dielectric rods or pores.

We will seek the solution of the system (8) numerically. The number of equations as well as the number of variables depends on the number of reciprocal 


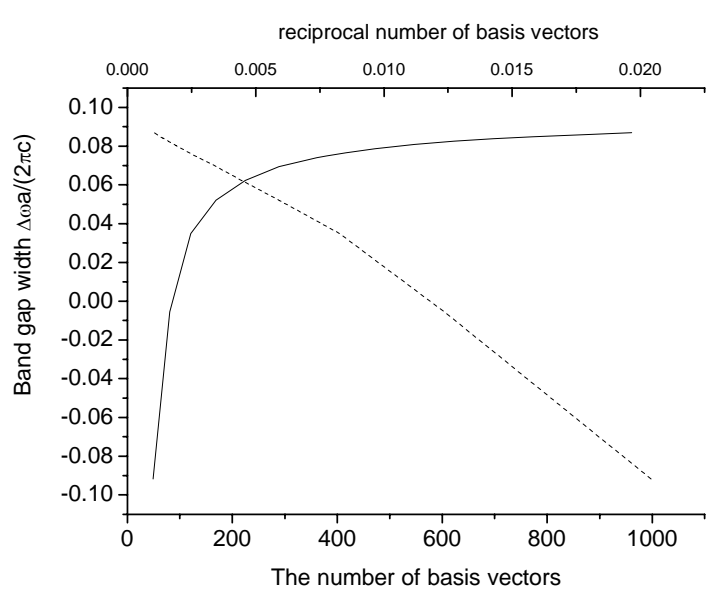

Fig. 2. Accuracy estimation. Solid line - the dependence of bandgap width vs the number of basis reciprocal vectors. The curve asymptotically tends to the "true" value of the bandgap at the infinite number of basis vectors. Dashed line $-1 / N$ dependence of the bandwidth.

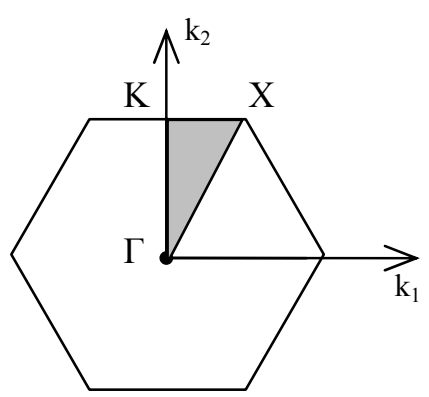

Fig. 3. The first Brilluoin zone for the triangular lattice.

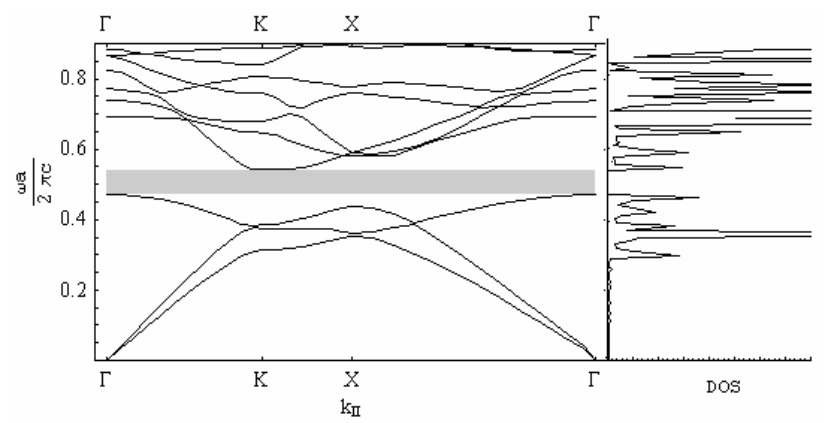

Fig. 4. The triangular two-dimensional photonic crystal. Inplane propagation.

Left side - the calculated photonic band structure, the right one - corresponding density of states, the shaded one -absolute photonic gap; $\varepsilon_{a}=1, \varepsilon_{b}=12, R=0.48 a, N=529$.

vectors that represents the basis of the Fourier expansion. We choose the reciprocal vectors occupying the parallelogram-shape area in reciprocal space with the origin of coordinates in the centre, and basis number $N=529$. The total number of equations in (8) is $3 N$. Because squares of frequency are only in the left side of
(8), we may represent the system as an eigenvalue problem of some matrix:

$\vec{A} \widehat{M}=\lambda \vec{A}$,

where $\vec{A}$ and $\lambda$ are the matrix $M$ eigenvectors and eigenvalues, respectively; $\lambda_{i}=\omega_{i}^{2} / c^{2}, i=1,2, \ldots, 3 N$; $\vec{A}=\left\{a_{1}\left(\vec{k}_{I I}+\vec{G}_{I I}\right) \ldots, a_{2}\left(\vec{k}_{I I}+\vec{G}_{I I}\right) \ldots, a_{3}\left(\vec{k}_{I I}+\vec{G}_{I I}\right)\right\}$.

Thus, by solving the eigenvalue problem (11) we can obtain the values of $\omega^{2} / c^{2}$ depending on the values of $\vec{k}_{I I}$, i.e, the dispersion law. For this purpose, the computer code in the Mathematica 5.0 package was created, and the numerical calculations were performed.

The size of the Fourier expansion basis $N$ is of great importance in the theory of photonic structures, and it is directly concerned with the accuracy of the plane-wave method. It is of interest what size of basis is sufficient for calculations and how the computational accuracy depends on the number of basis reciprocal vectors. Both questions should be the subject of investigation. Evidently, the physical results must be independent of the basis size. In order to estimate the behavior of dispersion curves under condition of $N \rightarrow \infty$, we have carried out the calculations for the $2 \mathrm{D}$ triangular photonic structure with $\varepsilon_{a}=1, \varepsilon=12, R=0.48 a$, for various numbers of reciprocal vectors $N$. Fig. 2 shows the dependence of the energy gap existing between the third and fourth photonic bands on the number of basis reciprocal vectors. It follows from Fig. 2 that the basis possessing less than 200 reciprocal vectors is quite insufficient taking into account that we do not know what is the limit of this curve if $N \rightarrow \infty$. By means of the Richardson criterion, we have estimated that the used basis $(N=529$ plane-waves) results in the calculation error that does not exceed $6 \%$. If we prolong the dashed curve in Fig. 2 to the left, the theoretical limit $N \rightarrow \infty$ of the energy gap will be reached using the Richardson criterion: $\Delta \approx 0.092$ in $\omega a / 2 \pi c$ units.

\section{Two-dimensional photonic crystal. The simple-wall case}

Let us consider the electromagnetic field propagating perpendicularly to pore axes in porous $2 \mathrm{D}$ photonic crystal with the triangular superlattice shown in Fig. 1. The primitive translation vectors are $\vec{a}_{1}=a(1,0)$, $\vec{a}_{2}=a\left(\frac{1}{2}, \frac{\sqrt{3}}{2}\right)$. According to (4), one can obtain the basis vectors of the reciprocal lattice $\vec{b}_{1}=2 \pi(1,-1 / \sqrt{3}) / a, \quad \vec{b}_{2}=2 \pi(0,2 / \sqrt{3}) / a \quad$ and, consequently, the first Brillouin zone (Fig. 3). The typical photonic band structure and density of states for 2D photonic crystal with the triangular superlattice are presented in Fig. 4. The calculation was performed with the basis number of reciprocal vectors $N=529$. The left 


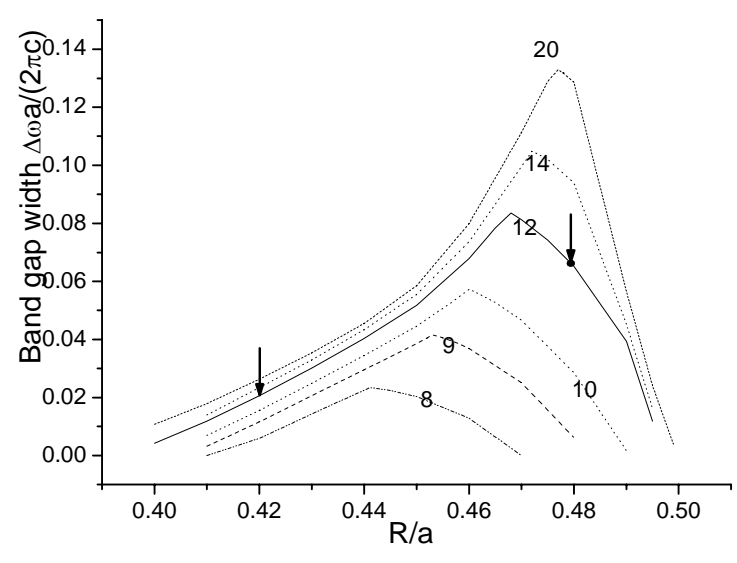

a)

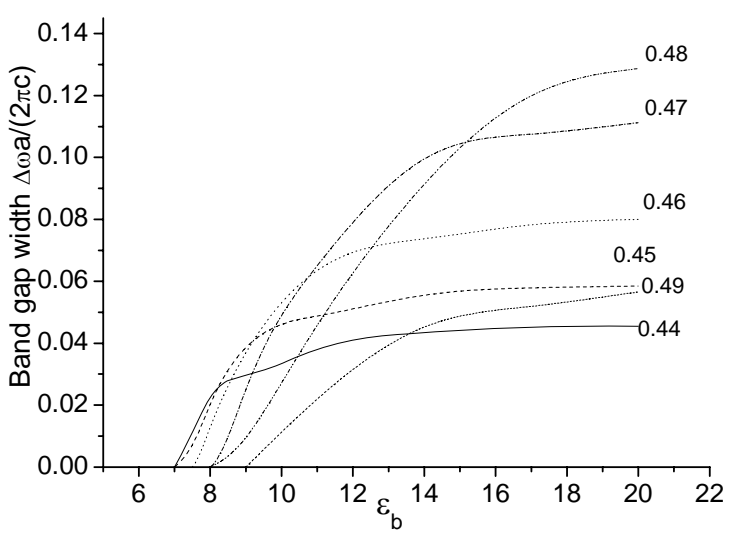

b)

Fig. 5. The lowest gap width behavior for $2 \mathrm{D}$ triangular lattice photonic crystal: $\mathrm{a}-\Delta \omega$ vs the pore radius for various medium dielectric constants denoted by magnitudes near each curve; $b-\Delta \omega$ vs the medium dielectric constant for various pore radii, values in $a$-unities near each curve. Arrows show the points in which $R=0.42 a$ and $R=0.48 a$ on the curve; $\varepsilon_{b}=12, \varepsilon_{a}=1$, $N=529$.

side of Fig. 4 shows the photonic band structure pattern along the $\Gamma-\mathrm{K}-\mathrm{X}-\Gamma$ way in the Brillouin zone for the first 10 branches. The lowest absolute energy gap is located between the third and fourth dispersion branches. On the curve of the density of photonic states (DOS) depicted in the right side of Fig. 4, the bandgap corresponds to the area with zero density. The $Y$-axis is the energy spectrum in non-dimensional frequencies $\omega a / 2 \pi c$, where $a$ is the lattice constant and $c$ is the light velocity. The horizontal axis represents the wavevectors running over $\Gamma-K, K-X$, and $X-\Gamma$ directions in the Brillouin zone (left, band structure pattern) and the density of photonic states in arbitrary units (right, DOS pattern).

The gap width depends on the ratio of dielectric constants and the pore radius (or filling fraction). In general, it depends on the angle of propagation, too. The plane-wave method describes electromagnetic waves in an infinite regular medium. An external boundary destroys the translational invariance. It is well known that the result of the boundary influence may be manifested in surface local modes having frequencies inside the gap. But the problem is to include a local imperfection into the plane-wave method based on the supposition that all field properties are periodical. Moreover, the plane boundary oriented in parallel to pore axes may be created by the infinite number of ways. Therefore, there exists a serious difficulty to connect the bandgap structure and other intrinsic mode parameters with that for an external source. Nevertheless, the chosen case of propagation perpendicular to the pore has a direct relation to the external incidence geometry for real macroporous photonic structures. Obviously, the generated by an external source electromagnetic waves that fall onto the crystal plane vertical interface perpendicularly to the pore axes will excite the intrinsic modes. The excited mode wavevectors should lie in the same plane and keep the same direction. One-, two- or multimode wavevectors corresponding to this incident wave may be obtained from Fig. 4 (left side) geometrically. Some more information may be provided by the shape of photonic branches and density of states. In particular, the high density areas correspond to the high transmittance, while the low densities correspond to the high reflection for external waves. The further consideration including reflected and transmitted waves intensities goes aside the framework of the plane-wave approach.

The width dependences for the gaps situated behind the third band on the pore radius for the photonic crystal with $\varepsilon_{a}=1$ and various $\varepsilon_{b}$ are depicted in Fig. 5a. All the curves have the main peak that corresponds to some optimal pore radius (filling fraction) for each structure. As follows from Fig. 5, if $\varepsilon_{a}=1, \varepsilon_{b}=8$ the maximal gap $\Delta \omega=0.023$ is reached when the pore radius value $R=0.441 a\left(f_{a}=0.7\right)$, while for the structure with $\varepsilon_{a}=1$, $\varepsilon_{b}=14$ the maximal gap $\Delta \omega=0.105$ appears when $R=0.472 a\left(f_{a}=0.81\right)$. It is of interest to compare the optimal filling fractions for photonic structures with different symmetry and geometry. Thus, 3D photonic crystal consisting of the air spheres embedded periodically in dielectric medium with the refractive index 3.6 and the diamond lattice symmetry has the maximal gap when the air filling fraction $f_{a}=0.81$. The similar structure for dielectric spheres placed in air gives the optimal air filling fraction $f_{a}=0.63$ [4]. For 3D layer-by-layer photonic structure consisting of the overlapping air cylinders inside the dielectric medium with the refractive index 3.6, the maximal gap was observed at $f_{a}=0.8$ [12]. And finally, our calculation made for 2D square lattice photonic crystals gives the optimal filling fraction $f_{a}=0.72$ for dielectric indices $\varepsilon_{a}=1, \varepsilon_{b}=12$.

Another interesting feature of curves depicted in Fig. $5 \mathrm{a}$ is that all of them end with falling areas in the 
vicinity of the limiting pore radius value $R=0.5 \mathrm{a}$. The gap decrease may be caused by diminution of an effective contrast between different parts of the photonic crystal elementary cell. Conversely, the bandgap growth area means that the effective contrast inside the elementary cell increases. It is necessary to note that the system properties change qualitatively at the value $R=a / 2$, since the periodic array of the circular pores in the medium transforms into the one of complicated shape columns in the air. One can conclude that the electromagnetic field "feels" this destruction of their symmetry.

The bandgap width is shown in Fig. 5b as a function of the dielectric constant $\varepsilon_{b}$ for various pore radii. The gap is opened for $\varepsilon_{b}>7$, and its value grows with increasing the contrast of dielectric constants, and finally, it saturates at $\varepsilon_{b}>20$. The most interesting behavior is demonstrated by the curve that corresponds to $R=0.49$ having the minimal index of growth up to $\varepsilon_{b}=14$. As it is clear from Fig. 5b, all the curves $0.45 \ldots 049$ correspond to the gap width growth, the curve 0.49 has the minimal index of the growth up to $\varepsilon_{b}=14$. To explain this fact, we should notice that at least a few points of this curve can be obtained from Fig. 5a by vertical crossing of the curves by the straight line $R=0.49$. Therefore, this curve really has lower values of bandgap width than, for example, the curve $R=0.48$.

The transmittance of electromagnetic waves propagating in 2D macroporous silicon structure parallel to macropores was investigated experimentally in [13]. There it was found that the reflection band is formed at the wavelength $\lambda=1.5 \lambda_{a}$ when $\varepsilon_{b}=13, \varepsilon_{a}=1$, and $f_{a}=0.84$. The optical period of the photonic structure $\lambda_{a}$ was defined as $\lambda_{a}=(a-2 R) \varepsilon_{a}{ }^{1 / 2}+2 R \varepsilon_{b}{ }^{1 / 2}$. The calculations made in [12] predicted the photonic bandgap formation which is common for $\mathrm{H}-$ and $\mathrm{E}$ polarization of electromagnetic irradiation at $\lambda \approx 1.2 \lambda_{a}$ when $\varepsilon_{b}=12.3, \varepsilon_{a}=1$, and $f_{a}=0.73$.

In the case presented in Fig. 4 for perpendicular incidence and $a=1 \mu \mathrm{m}$, we have $\lambda_{a}=1099 \mathrm{~nm}$, the band occupies position between $\lambda=1851$ and $2127 \mathrm{~nm}$. To obtain the photonic crystal with maximal gap width for the assumed lattice constant and dielectric constants $\varepsilon_{b}=12, \varepsilon_{a}=1$, we must create the structure with the following parameters: $a=1 \mu \mathrm{m}, R=470 \mathrm{~nm}$, and $l=60 \mathrm{~nm}$, where $l=a-2 R$ is the shortest distance between the pore edges. In this case $\left(\Delta \omega=0.081 \omega_{0}\right)$, the spectral width of the gap will be $\Delta \lambda \approx 345 \mathrm{~nm}$. As the light sources have a spectral width of radiation, the gap width must be many times more than the spectral width. This is important if used femtosecond lasers have comparatively large (up to $10 \mathrm{~nm}$ ) spectral width of radiation.

The 2D photonic crystals were intensively investigated both theoretically and experimentally in a plethora of works. Nevertheless, in most cases, only the "simple" 2D photonic crystals were considered. Further, we will calculate some more complicated 2D photonic structures containing lamellar pore walls.

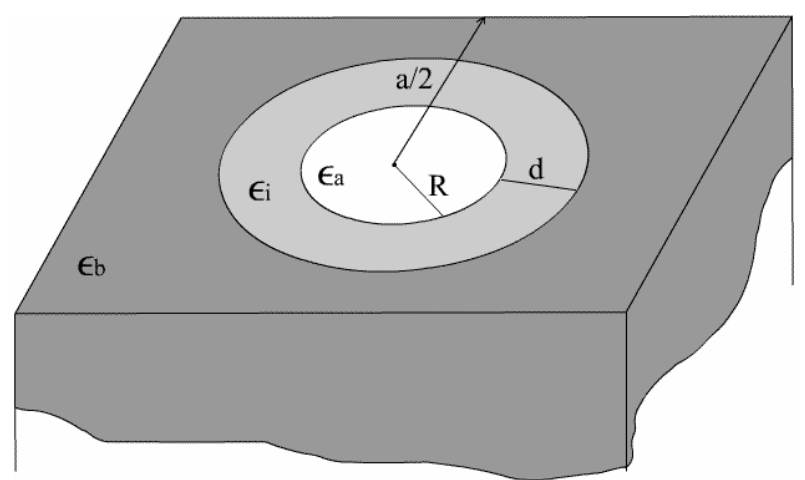

Fig. 6. The unit cell of the $2 \mathrm{D}$ photonic crystal with surface interlayer. $\varepsilon_{i}$ and $d$ are the dielectric constant and the thickness of the interlayer, respectively; $R$ is the pore radius; $\varepsilon_{a}=1$, $\varepsilon_{b}=12$.

\section{Two-dimensional photonic structure with layered pore surfaces}

Electrochemical etching is one of the most efficient ways to fabricate silicon macropore structures [14, 15]. Depending on the parameters of etching process, the sample area near the pore intrinsic surface being the ionic "battle field" becomes different from the bulk. It means that there arise the surface interlayers inside pores that may be of various thicknesses, dielectric properties, densities of charge carriers, and so on. Therefore, we consider the photonic crystals with the complicate structure of inner macropore surface. Another possible interesting application of such structures emerges when the additional interlayer has the optical non-linearity. In this case, the optical properties of photonic structures depend on the electromagnetic wave intensity. Thus, by varying the irradiation intensity we will be able to operate by the optical signal if the wavelength lies near the photonic bandgap edge [16].

The unit cell of the system under consideration is presented in Fig. 6. Here, $\varepsilon_{i}, \varepsilon_{a}, \varepsilon_{b}$ are the dielectric constants of the surface interlayer, pore, and rest of medium, respectively, $d$ is the thickness of the interlayer, $R$ is the intrinsic radius of the pore. Evidently, the additional interlayer does not change the general form of equations (8) and the shape of the first Brillouin zone (Fig. 3), since the periodicity of the system is not changed. The presence of the surface layer affects the coordinate dependence of the dielectric function. Consequently, the form of the dielectric function Fourier components $\widehat{\kappa}$ will be changed. So, for the photonic crystal with the unit cell geometry presented in Fig. 6, we have obtained the following expression for the Fourier components of the dielectric function:

$$
\hat{\kappa}\left(G_{I I}\right)=\left\{\begin{array}{l}
\frac{2 \cdot\left(\frac{1}{\varepsilon_{a}}+\frac{1}{\varepsilon_{b}}\right) \cdot f_{1} J_{1}\left(G_{I I} R\right)}{G_{I I} R}+\frac{2 \cdot\left(\frac{1}{\varepsilon_{i}}-\frac{1}{\varepsilon_{b}}\right) \cdot f_{3} J_{1}\left(G_{I I} \cdot(R+d)\right)}{G_{I I}(R+d)}, G_{I I} \neq 0 \\
\left(\frac{f_{1}}{\varepsilon_{a}}+\frac{f_{2}}{\varepsilon_{b}}+\frac{f_{i}}{\varepsilon_{i}}\right), G_{I I}=0
\end{array}\right.
$$




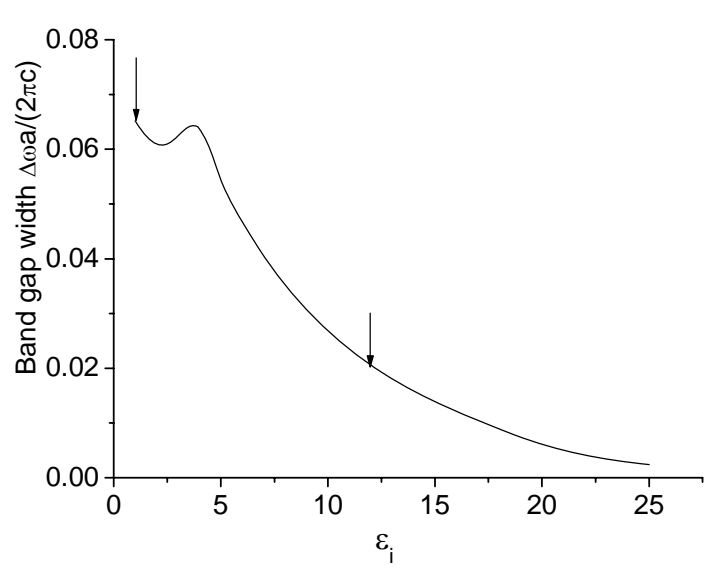

Fig. 7. Bandgap width vs the interlayer dielectric constant dependence. The arrows show the points where three-medium system transforms into the two-medium one, namely, when $\varepsilon_{i}=1$ and $\varepsilon_{i}=12 . R=0.42 a, d=0.06 a$.

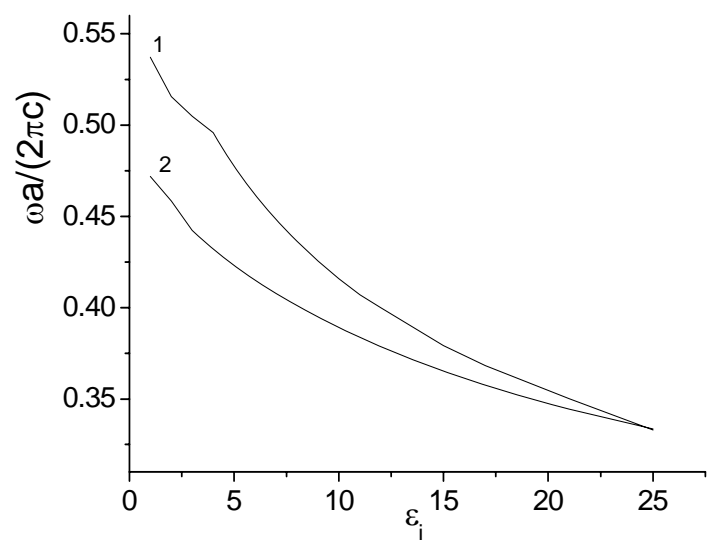

Fig. 8. The bandgap edge shift with increasing $\varepsilon_{i}$. Curve 1 represents the upper edge, curve $2-$ the lower one. $R=0.42 a$, $d=0.06 a, N=529$.

where $\quad f_{1}=\frac{2 \pi}{\sqrt{3}} \frac{R^{2}}{a^{2}}, \quad f_{i}=\frac{2 \pi}{\sqrt{3}} \frac{\left((R+d)^{2}-R^{2}\right)}{a^{2}}$, $f_{3}=\frac{2 \pi}{\sqrt{3}} \frac{(R+d)^{2}}{a^{2}}, f_{2}=1-f_{1}-f_{i}$, and $J_{1}(x)$ is the Bessel function. It is necessary to note that the expression (12) transforms into (9) in limit cases $\varepsilon_{i}=\varepsilon_{a}$, $\varepsilon_{i}=\varepsilon_{b}, d=0, R=0$. We will analyze the photonic band structure dependence on the interlayer dielectric constant $\varepsilon_{i}$ and interlayer thickness $d$. For simplification of further computations, we fix the dielectric constants at the values $\varepsilon_{a}=1, \varepsilon_{b}=12$ and consider only the cases which possess a gap in the band structure according to Fig. 5 . For example, we can conclude that if the sum $R+d$ will be less than $0.4 a$ the gap will not exist for any $\varepsilon_{i}$ values in the interval $\varepsilon_{i} \in(1 ; 12)$. This follows from the fact that the case $\varepsilon_{i}=\varepsilon_{a}=1$ corresponds to the "usual" photonic crystal with the pore radius $(R+d)$ in the medium with the dielectric constant $\varepsilon_{b}=12$, while the case $\varepsilon_{i}=\varepsilon_{b}=12$ corresponds to the "usual" photonic crystal with the pore radius $R$ in the medium with dielectric constant $\varepsilon_{b}=12$. Then, taking into account the dependence depicted in Fig. 5, we conclude that, for the system with $\varepsilon_{a}=1$ and $\varepsilon_{b}=12$, the gap appearance condition is determined by the expression $R+d>0.4 a$.

We have calculated in-plane propagation of electromagnetic waves through the triangular lattice 2D photonic crystal with lamellar pore walls. In Fig. 7, there is the width dependence of the gap that is located between the third and fourth dispersion branches (see Fig. 4) on the interlayer dielectric constant. The system has the following parameters: $R=0.42 a, d=0.06 a$, $\varepsilon_{a}=1 ; \varepsilon_{b}=12, f_{a}=0.38, f_{i}=0.34$, where $f_{a}$ and $f_{i}$ are the fractions filled by air and the interlayer ones, respectively.

As was denoted earlier, the gap width and bandgap edge position depend on the dielectric constant contrast, filling fractions and system symmetry. We believe that some effective quantity exists which includes all these three factors. This parameter should define both the ability of a photonic structure to possess the photonic gap and optimal relations between system parameters to obtain the wider bandgap. Let us call this parameter by photonic contrast. For example, we can conclude from Fig. 5a that, for two-medium structure with $\varepsilon_{a}=1$, $\varepsilon_{b}=12$, the maximal photonic contrast is achieved when $f_{a}=0.79$. For the similar structure with $\varepsilon_{a}=1, \varepsilon_{b}=20$, the peak arises at $f_{a}=0.83$.

Further, we will try to describe the behavior of the lamellar pore structures by using the effective photonic contrast mentioned above. The arrows in Fig. 7 show two points where the three-medium system transforms into the two-medium one, just when $\varepsilon_{i}=1$ and $\varepsilon_{i}=12$. The same points are also present in Fig. 5a, and we can see that, between these two points, the photonic contrast of the two-medium system has the maximum value. Thus, we assume that the three-medium structure would have also the peak between two points. But in Fig. 7, the curve decreases initially and then tends to a peak. A consequence of the above is the fact that the third medium introducing decreases the photonic contrast of the system. Another circumstance that confirms this conclusion is that all examined three-component photonic crystals acquire the wider gap when they were transformed into the two-component ones.

To obtain the formal expression describing the photonic contrast, the four variables gap function should be built as a hyper-surface in 5-dimensional space of variables $\Delta \omega, \varepsilon_{b}, \varepsilon_{i}, d, R$

After that, the every section of the gap function surface $\Delta \omega=S\left(\varepsilon_{b}, \varepsilon_{i}, d, R\right)$ matching the maximum and characterizing by monotonous growth from $\Delta \omega=0$ to $\Delta \omega=\max$ may be used to represent the photonic 


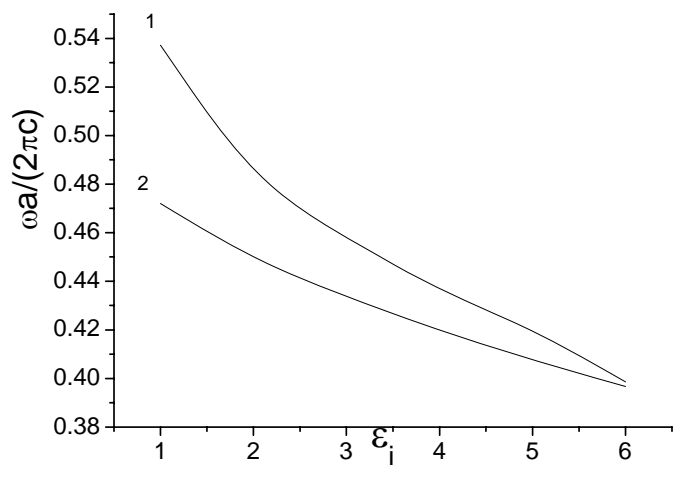

a)

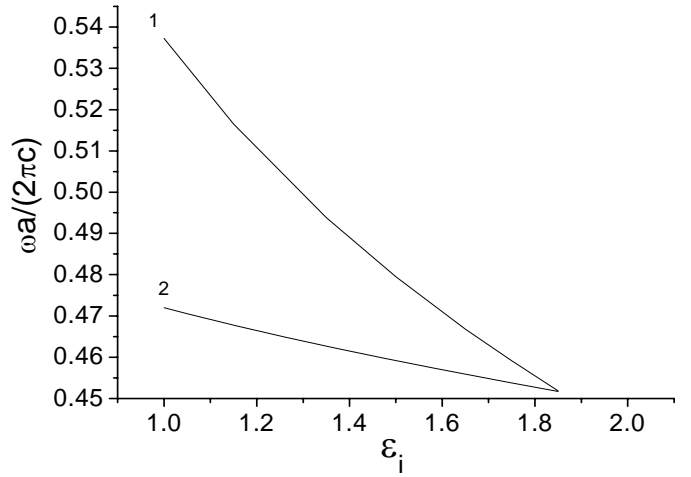

b)

Fig. 9. Shifts of the bandgap edges with increasing $\varepsilon_{i}: \mathrm{a}-R=0.35 a, d=0.13 a, f_{a}=0.38, f_{i}=0.34, \varepsilon_{a}=1, \varepsilon_{b}=12 ; \mathrm{b}-R=0.25 a$, $d=0.23 a, f_{a}=0.19, f_{i}=0.52, \varepsilon_{a}=1 ; \varepsilon_{b}=12$. Curve 1 represents the upper edge, curve 2 - the lower one.

contrast. There exist many ways beginning with the 4dimensional point $\varepsilon_{b 0}, \varepsilon_{i 0}, d_{0}, R_{0}$ where $\Delta \omega=0$ and finishing in the point $\varepsilon_{b m}, \varepsilon_{i m}, d_{m}, R_{m}$ where $\Delta \omega=\max$. All of them may be described by a special monotonous function $C_{t}$

$$
C_{t}=\frac{1}{4}\left[\begin{array}{l}
\left(\frac{\varepsilon_{b}-\varepsilon_{b 0}}{\varepsilon_{b m}-\varepsilon_{b 0}}\right)^{2}+\left(\frac{\varepsilon_{i}-\varepsilon_{i 0}}{\varepsilon_{i m}-\varepsilon_{i 0}}\right)^{2}+ \\
+\left(\frac{d-d_{0}}{d_{m}-d_{0}}\right)^{2}+\left(\frac{R-R_{0}}{R_{m}-R_{0}}\right)^{2}
\end{array}\right]
$$

that presents the photonic contrast of the system. The photonic contrast function $C_{t}$ is positively defined, and it ranges from 0 to 1 for the chosen area of parameters in the vicinity of $\Delta \omega=\max$. The coefficient $1 / 4$ in (13) is caused by the hyper-surface dimension. For example, the simplewall case considered in the previous section is described by two terms in (13) and the coefficient is $1 / 2$. Then, the left arrow in Fig. 5a corresponds to the photonic contrast $C_{t}=0.066$ and, for the right arrow, we have $C_{t}=0.56$. In the general case presented in Fig. 9, the calculated by (13) photonic contrast is $C_{t}=0.090$ when $\varepsilon_{i}=3$ (Fig. 9a) and $C_{t}=0.063$ when $\varepsilon_{i}=1.5$ (Fig. 9b).

The shift of the bandgap higher and lower edges is shown in Fig. 8. As it follows from the figure, the gap edges are shifted into the low-frequency range with increasing $\varepsilon_{i}$. To explain the effect, let us analyze the system (8). The squares of unknown frequencies $\omega$ in the right side are inversely proportional to the Fourier coefficients $\widehat{\kappa}\left(\vec{G}_{I I}-\vec{G}_{I I}^{\prime}\right)$ of the dielectric function. Consequently, if $\widehat{\kappa}\left(\vec{G}_{I I}-\vec{G}_{I I}^{\prime}\right)$, for example, is doubled, the frequency $\omega$ reduces by a factor of the square root of two. Therefore, the ordinate axis "scale" will be decreased by a factor of $\sqrt{2}$. To confirm this conclusion, we have compared the results for two photonic crystals with similar geometry when the first of them had the dielectric constants $\varepsilon_{a}=1, \varepsilon_{b}=9$ and the second one had $\varepsilon_{a}=2, \varepsilon_{b}=18$. All the frequency characteristic values (gap width, gap edges) really differed by $\sqrt{2}$ times.

The bandgap edge shift dependences for the structures with $R=0.35 a, d=0.13 a, f_{a}=0.38, f_{i}=0.34$, and $R=0.25 a, d=0.23 a, f_{a}=0.19, f_{i}=0.52$ are shown in Figs 9a and b, respectively. The gap edge behavior is similar to the previous case, but the bandgap vanishes when the intermediate layer dielectric function values $\varepsilon_{i} \approx 6.2$ (Fig. 9a) and $\varepsilon_{i} \approx 1.87$ (Fig. 9b). In the latter case, the main role in band structure formation is played neither by air, nor by medium. It is done by the interlayer material, since it fills now more than a half of the total volume. Therefore, varying the interlayer dielectric constant twice less than results in bandgap vanishing. On the contrary, the interlayer thickness decrease must result in the reduction of its influence on the photonic band structure. Really, in the case when $R=0.46 a, d=0.01 a$, the gap width varies only in the interval $(0.082 \ldots 0.066)$ with $\varepsilon_{i}$ increasing from 1 to 20 .

\section{Conclusion}

We have considered a possibility to vary the lowest gap width and this gap position in $2 \mathrm{D}$ photonic crystals by introducing the additional interlayers on pore surfaces. The bandgap width and bandgap edge position dependences on the interlayer dielectric constant for various system parameters $R, d$, and $\varepsilon_{i}$ at the fixed values $\varepsilon_{a}=1$ and $\varepsilon_{b}=12$ have been calculated. The analysis results in the following:

- the introduction of additional interlayers decreases the photonic bandgap for given geometry;

- the photonic gap shifts to the low frequencies, and its width decreases if $\varepsilon_{i}$ is increased;

- the gap vanishes for high values of the interlayer thickness $d>0.2 a$ for the low values of the interlayer dielectric constant $\varepsilon_{i}$. 
The technology of macroporous silicon structures fabrication is intensively developed during the last decade. The structures with lattice constants $a=1$ to $15 \mu \mathrm{m}$ and pore radii $R=0.3$ to $10 \mu \mathrm{m}$ were obtained in $[14,15,17,18]$. We suppose that the complex structure of the pore walls theoretically predicted in the work may be realized by the existing experimental methods.

\section{Acknowledgements}

This work was supported by the grant STCU-2444.

\section{References}

1. E. Yablonovich, Inhibited spontaneous emission in solid-state physics and electronics // Phys Rev. Lett. 58, p. 2059-2062 (1987); E. Yablonovitch, T.J. Gmitter, and K.M. Leung, Photonic band structure: The face-centered-cubic case // Ibid. 63, p. 1950-1953 (1991).

2. S. John, Strong localizations of photons in certain disordered dielectric super lattices // Phys Rev. Lett. 58, p. 2486-2489 (1987); S. John and R. Rangavajan // Phys Rev.B 38, p. 10101 (1988).

3. J.D. Joannopoulos, R.D. Meade and J.N. Winn, Photonic Crystals, Princeton University Press, Princeton (1995).

4. K.M. Ho, C.T. Chan, and C.M. Soukoulis, Existence of a photonic band gap in periodic dielectric structure // Phys Rev. Lett. 65, p. 31523156 (1990).

5. R.D. Meade, K.D. Brommer, A.M. Rappe, and J.D. Joannopoulos, Existence of a photonic bandgap in two dimensions // Appl. Phys. Lett. 61, p. 495-499 (1992).

6. E.N. Economou and A. Zdetsis, Classical wave propagation in periodic structures // Phys.Rev.B 40, p. 1334 (1989).

7. Ze Shang and S. Satpathy, Electromagnetic wave propagation in periodic structures: Bloch wave solutions of Maxwell's equations // Phys Rev. Lett. 65, p. 2650-2652 (1990).

8. K.M. Leung and Y.F. Liu, Full vector-wave calculation of photonic band structures in face- centered-cubic dielectric media // Ibid. 65, p. 2646 (1990).

9. P.R. Villeneuve, M. Piche, Photonic band gaps in two-dimensional square and hexagonal lattices // Phys. Rev. B 46, p. 4969-4972 (1992).

10. A.A. Maradudin and A.R. McGurn, Out-of-plane propagation of electromagnetic waves in a twodimensional periodic dielectric medium // J. Mod. Opt. 41, p. 275 (1994).

11. W.M. Robertson, G. Arjavalingam, R.D. Meade, K.D. Brommer, A.M. Rapper, J.D. Joannopoulos, Measurement of photonic band structure in a twodimensional periodic dielectric array // Phys. Rev. Lett. 68, p. 2023-2026 (1992).

12. R. Biswas, C.T. Chan, M. Sigalas, C.M. Soukolis, and K.M. Ho, Photonic bandgap materials // Photonic bandgap materials (NATO ASI Series, ed. by M. Soukoulis), Series E, Vol. 315, p. 23-40 (1996).

13. L.A. Karachevtseva, O.A. Lytvynenko, E.A. Malovichko, O.I. Stronska, Optical transmittance of macroporous silicon structures // Semiconductor Physics, Quantum Electronics \& Optoelectronics 4(4), p. 347 (2001).

14. V. Lehmann, H. Foll, Formation mechanism and properties of electrochemically etched trenches in $n$ type silicon // J. Electrochem. Soc. 137(2), p. 653659 (1990).

15. U. Gruning, V. Lehmann, S. Ottow, K. Bushch // Ibid. 146, p. 385 (1996).

16. E.Ya. Glushko, All-optical signal processing in photonic structures with nonlinearity // Opt. Communs 242, in press (2005).

17. E.V. Astrova, T.N. Borovitskaya, V.A. Tolmachev, T.S. Petrova, Quartz microtubes on the base of macroporous silicon // Fiz. Tekhn. Poluprov. 38, p. 1125-1129 (2004).

18. E.V. Astrova, T.N. Borovitskaya, T.S. Petrova, M.V. Zamoryanskaya, The picture creation technology for macroporous silicon and producing of 2D photonic crystals with vertical walls // Ibid. 38, p. 1121-1124 (2004). 